\title{
The effect of tidal locking on the magnetospheric and atmospheric evolution of "Hot Jupiters"
}

\author{
J.-M. Grießmeier ${ }^{1}$, A. Stadelmann ${ }^{2}$, T. Penz ${ }^{3,4}$, H. Lammer ${ }^{4}$, F. Selsis ${ }^{5}$, I. Ribas ${ }^{6,7}$, \\ E. F. Guinan ${ }^{8}$, U. Motschmann ${ }^{1}$, H. K. Biernat ${ }^{4,3}$, and W. W. Weiss ${ }^{9}$
}

1 Institut für Theoretische Physik, Technische Universität Braunschweig, Mendelssohnstraße 3, 38106 Braunschweig, Germany

e-mail: j-m.griessmeier@tu-bs.de; u.motschmann@tu-bs.de

2 Institut für Geophysik und extraterrestrische Physik, Technische Universität Braunschweig, Mendelssohnstraße 3, 38106 Braunschweig, Germany e-mail: a.stadelmann@tu-bs.de

3 Institute for Theoretical Physics, University of Graz, Universitätsplatz 5, 8010 Graz, Austria e-mail: penzt@stud.uni-graz.at

${ }^{4}$ Space Research Institute, Austrian Academy of Sciences, Schmiedlstrasse 6, 8042 Graz, Austria e-mail: helmut.lammer@oeaw.ac.at; helfried.biernat@oeaw.ac.at

5 Centro de Astrobiología (CSIC-INTA), Carretera de Ajalvir, km 428850 Torrejon de Ardoz, Madrid, Spain e-mail: selsisf@inta.es; selsis@obs.u-bordeaux1.fr

${ }^{6}$ Institut de Ciències de l'Espai (CSIC-IEEC), c/Gran Capità, 2-4, 08034 Barcelona, Spain e-mail: iribas@ieec.fcr.es

7 Departament d' Astronomia i Meteorologia, Universitat de Barcelona, Av. Diagonal 647, 08028 Barcelona, Spain

8 Department of Astronomy and Astrophysics, Villanova University, Villanova, PA 19085, USA e-mail: edward.guinan@villanova.edu

9 Department for Astronomy, University of Vienna, Türkenschanzstrasse 17, 1180 Vienna, Austria

e-mail: weiss@astro.univie.ac.at

Received 14 November 2003 / Accepted 14 June 2004

\begin{abstract}
We study the interaction between the planetary magnetosphere and atmosphere of the close-in extrasolar planets HD 209458b and OGLE-TR-56b with the stellar wind during the evolution of their host stars. Recent astrophysical observations of solar-like stars indicate that the radiation and particle environments of young stars are orders of magnitudes larger than for stars with ages comparable to the sun $(\sim 4.6 \mathrm{Gyr})$. We model the interaction for the present and for early evolutionary stages, showing that it is possible that "Hot Jupiters" have an ionosphere-stellar wind interaction like Venus. Our study suggests that the internal magnetic field of exoplanets orbiting close to their host stars may be very weak due to tidal locking. The magnetic moments can be less than one tenth of the value presently observed for the rapidly rotating planet Jupiter. We find that the stronger stellar wind of younger solar-type stars compresses the magnetosphere to a standoff distance at which the ionized part of the upper atmosphere, hydrodynamically expanded by the XUV-flux, builds an obstacle. Because of a much larger stellar wind particle flux during the first $\sim 0.5$ Gyr after the host stars arrived on the Zero-Age-Main-Sequence, "Hot Jupiters" may have not been protected by their intrinsic magnetic fields, even if one neglects the effect of tidal locking. In such a case, the unshielded upper atmosphere will be affected by different ionization and non-thermal ion loss processes. This contributes to the estimated neutral hydrogen loss rates of about $\geq 10^{10} \mathrm{~g} / \mathrm{s}$ of the observed expanded exosphere of HD 209458b (Vidal-Madjar et al. 2003) and will be an ionized part of the estimated upper energy-limited neutral hydrogen loss rates of about $10^{12} \mathrm{~g} / \mathrm{s}$ (Lammer et al. 2003a).
\end{abstract}

Key words. stars: planetary systems - stars: winds, outflows - magnetic fields

\section{Introduction}

Since 1995, more than 100 Jupiter-class exoplanets were detected ${ }^{1}$, many of them in close orbits around $\mathrm{G}$ and $\mathrm{K}$ stars. Two of these planets are HD 209458b and OGLE-TR-56b, and they orbit their hosts stars at $0.045 \mathrm{AU}$ and $0.0225 \mathrm{AU}$

1 See e.g. The Extrasolar Planets Encyclopaedia on http://www.obspm.fr/encycl/encycl.html respectively. They are known to be gas giants with hydrogen as their main constituent (Burrows et al. 2000; Sasselov 2003). These gas giants in an orbit very close to their parent star present a very special class of planets and will be referred to as "Hot Jupiters" throughout this study.

Besides the strong stellar irradiation, one of the special features of these planets is their low rotation rate. This results from tidal locking due to gravitational dissipation: the time scale 
for synchronous rotation depends on the orbital radius $d$ such as $\tau_{\text {sync }}=O\left(d^{6}\right)$ (Goldreich \& Soter 1966). Thus a planet at $0.1 \mathrm{AU}$ will be tidally locked after much shorter time than a planet of comparable composition at $1 \mathrm{AU}$ or beyond. The reduced rotation rate has severe implications for the planetary magnetic moment (due to a weaker dynamo-effect), resulting in comparatively weak magnetic fields and small magnetospheres. For the calculation of the magnetic moment, a new and more realistic approach for the determination of the size of the dynamo-region is given. These calculations indicate even smaller magnetic moments than the usual estimates. The small size of the magnetosphere will in turn contribute to enhanced particle loss.

Recent Hubble Space Telescope (HST) observations of the upper atmosphere expanding out to about three planetary radii as well as neutral hydrogen escape rates larger than about $10^{10} \mathrm{~g} / \mathrm{s}$ have been found for HD 209458b (Vidal-Madjar et al. 2003, 2004). This indicates that XUV-driven hydrodynamic conditions are relevant for "Hot Jupiters". Guillot et al. (1996) roughly estimated the total (ion and neutral gas) loss for hydrogen. The obtained value of about $10^{10} \mathrm{~g} / \mathrm{s}$ is compatible with the lower limit of the observed values. We, however, suggest that it is hydrodynamical expansion rather than these nonthermal loss processes which are responsible for the observed expansion of the upper atmosphere. In recent studies of close-in giant exoplanets, the radiative effective temperature (which is not physically relevant for atmospheric escape processes or atmospheric expansion) was used to estimate atmospheric thermal loss rates (Konacki et al. 2003; Sasselov 2003). Rather than the equilibrium temperature, it is the exospheric temperature which is decisive for these processes. Therefore, these studies lead to significant underestimations of thermal loss rates and to conclusions of long-term atmospheric stability.

In a recent study Lammer et al. (2003a) estimated the exospheric temperature of "Hot Jupiters" by assuming that they have hydrogen rich upper atmospheres which are heated by the absorption of X-rays and extreme ultraviolet (XUV) radiation (Bauer 1971; Gross 1972; Watson et al. 1981). Lammer et al. (2003a) found that exospheric temperatures of the order of $10^{4} \mathrm{~K}$ may develop at orbital distances $<0.2 \mathrm{AU}$. In this case the Jeans approach fails and XUV-driven hydrodynamic atmospheric expansion as well as energy or diffusion limited escape must be considered. Because of high XUV-driven loss rates and atmospheric expansion, the temperature can decrease to values comparable to the effective temperature once the upper atmosphere reaches hydrodynamic conditions. The study agrees well with the observations of Vidal-Madjar et al. (2003, 2004) and results in moving the atmospheric barrier closer to the magnetospheric boundary.

The aim of this paper is to study whether the magnetic moments of "Hot Jupiters" produce magnetospheres which are extensive enough to protect the planet's expanded upper atmospheres. Otherwise, Venus-like ion loss processes will be caused by the incoming stellar wind. In Sect. 2 we introduce some basic properties of exoplanets required in the following and examine the effect of tidal locking on the magnetic moments of close-in exoplanets. Section 3 deals with the evolution of the radiation (Sect. 3.1) and particle environment (Sect. 3.2) of solar-like stars over their life-time. In Sect. 4 results from Sects. 2 and 3.2 are used to model the interaction of the stellar wind with the magnetosphere. The magnetospheric standoff distances of HD 209458b and OGLE-TR-56b are calculated for the conditions valid for a stellar system of an age comparable to the solar system (4.6 Gyr) and also for a younger stellar system $(0.5$ Gyr). Finally, in Sect. 5 we use the results of Sect. 4 to calculate the expansion of the upper atmospheres and also discuss the atmospheric protection by intrinsic magnetic fields, ion erosion and the implications for the evolution of "Hot Jupiters".

\section{Effect of tidal locking on planetary magnetic moments}

To properly estimate the size of the magnetosphere (Sect. 4) and the effects of atmospheric expansion and stellar wind atmosphere interaction in Sect. 5 , it is necessary to know the basic properties of the planet. The planetary mass and radius can be obtained directly from observational data. Another fundamental property of the planet that is needed in this context is the value of the planetary magnetic dipole moment. Recently, the chromospheric activity of the planet-hosting star HD 179949 was suggested as a first tentative proof for the existence of exomagnetospheres (Shkolnik et al. 2003). However, at this time no further information on the strengths of exosolar planetary magnetic fields can be obtained.

Fortunately, there are several analytical models that permit an estimation of the planetary magnetic dipole moment parallel to the rotation axis. These models yield the following scaling laws:

$\mathcal{M} \propto \quad \rho_{\mathrm{c}}^{1 / 2} \omega r_{\mathrm{c}}^{4}$

(Busse 1976)

$\mathcal{M} \propto \rho_{\mathrm{c}}^{1 / 2} \omega^{1 / 2} r_{\mathrm{c}}^{3} \sigma^{-1 / 2} \quad$ (Stevenson 1983, dissipative case)(2)

$\mathcal{M} \propto \rho_{\mathrm{c}}^{1 / 2} \omega^{3 / 4} r_{\mathrm{c}}^{7 / 2} \sigma^{-1 / 4} \quad$ (Mizutani et al. 1992)

$\mathcal{M} \propto \rho_{\mathrm{c}}^{1 / 2} \omega^{1 / 2} r_{\mathrm{c}}^{3} \sigma^{-1 / 2} \quad$ (Mizutani et al. 1992)

$\mathcal{M} \propto \quad \rho_{\mathrm{c}}^{1 / 2} \omega r_{\mathrm{c}}^{7 / 2}$

(Sano 1993)

where $\mathcal{M}$ is the planetary magnetic dipole moment, $r_{\mathrm{c}}$ the radius of the dynamo-region (frequently also called the "core radius") and $\omega$ the velocity of rotation of the planet around its axis. In the dynamo region the mass density is $\rho_{\mathrm{c}}$ and the conductivity is $\sigma$. At least two further scaling laws exist, but they are of no relevance in this context: the first scaling ("Blackett's law") originally proposed by Blackett (1947) was based on a hypothesis that was later disproved experimentally. As a result, the theory was withdrawn (Blackett 1952). The scaling used by Curtis \& Ness (1986) requires the heat flux arising from internal sources of the planet. This is an observable quantity in the case of planets in the solar system, but not for extrasolar planets. For close-in extrasolar giant planets highly different conditions are expected (i.e. strong external heating by stellar irradiation). Thus it is difficult to estimate the heat flux arising from sources in the dynamo-region of these planets from solar sytem data.

It is clear from Eqs. (1) to (5) that the magnetic moment $\mathcal{M}$ is determined by the internal properties of a planet (i.e. the properties of the dynamo region $r_{\mathrm{c}}, \rho_{\mathrm{c}}$ ) and not so much by the external parameters which are much easier to measure (like 
the planetary mass or radius). To overcome this problem, Curtis \& Ness (1986) use an empirical scaling law to determine the core radius $r_{\mathrm{c}}$ as a function of the planetary mass $M$. The best fit to our solar system (using the planets Mercury, Earth, Jupiter and Saturn) is found to be:

$r_{\mathrm{c}} \propto M^{0.44}$,

where $M$ is the mass of the planet. In this approach the core radius only depends on the mass of the planet. This seems reasonable for planets which have evolved to some equilibrium state, i.e. for which the radius $R$ is well determined by the mass $M$. For young planets, where for a given mass the radius is a function of the age, mass $M$ and radius $R$ should better both be considered in a scaling law of the form

$r_{\mathrm{c}} \propto M^{\alpha} R^{\beta}$.

This situation is typical for "Hot Jupiters", which have densities significantly lower than Jupiter. For this increased radius, different explanations are suggested. These include downward transport of kinetic energy generated by the intense stellar irradiation (Guillot \& Showman 2002; Bodenheimer et al. 2003), or dissipation of energy arising from tidal circularisation (Bodenheimer et al. 2003). Normalizing $r_{\mathrm{c}}, M$ and $R$ to the respective values of Jupiter and using the best fit for the planets Saturn, Uranus and Neptune to Eq. (7), one obtains

$r_{\mathrm{c}} \propto M^{0.75} R^{-0.96}$.

For this fit, the following values were adopted: $r_{\mathrm{c}}=0.9 R$ for Jupiter and $r_{\mathrm{c}}=0.5 R$ for Saturn (Nellis 2000), $r_{\mathrm{c}}=18000 \mathrm{~km}$ (i.e. $r_{\mathrm{c}}=0.7 R$ ) and $r_{\mathrm{c}}=19000 \mathrm{~km}$ (i.e. $r_{\mathrm{c}}=0.75 R$ ) for Uranus and Neptune (Hubbard 1984).

Relation (8) yields the correct behaviour. On the one hand, if the radius is kept constant, increasing mass will lead to a larger core. On the other hand, keeping the mass constant and increasing the radius will decrease the average density, and the volume of high enough density for dynamo generation will also decrease. Similarly, for a fixed average density (i.e. $R \propto M^{1 / 3}$ ), increasing the mass increases the core radius.

Of course, relations (6) and (8) only have a physical meaning if $r_{\mathrm{c}}<R$ is fulfilled. For Eq. (6) this is not the case if $M>2.6 M_{\mathrm{J}}$. This is satisfied for the two planets considered here. To demonstrate that in these cases Eq. (8) does not lead to unrealistic parameters, the core radii resulting from Eq. (8), normalized to the planetary radius, are given in Table 1 . Note that Eqs. (6) and (8) do not include the effect of stellar heating. For "Hot Jupiters" the large temperatures could lead to additional ionization, thus slightly increasing the core radius $r_{\mathrm{c}}$ and the magnetic moment $\mathcal{M}$.

Inserting Eqs. (6) or (8) into the scalings (1) to (5) the dipole moment can be expressed in terms of planetary parameters for which reasonable estimates exist. For the planets HD 209458b and OGLE-TR-56b we adopt the values given in Table 1. Most of the quantities in Table 1 are given in units normalized to Jupiter (denoted by subscript $\mathrm{J}$ ), with $R_{\mathrm{J}}=$ $71490 \mathrm{~km}, M_{\mathrm{J}}=1.9 \times 10^{27} \mathrm{~kg}, \omega_{\mathrm{J}}=1.76 \times 10^{-4} \mathrm{~s}^{-1}$, $\mathcal{M}_{\mathrm{J}}=1.5 \times 10^{27} \mathrm{Am}^{2}$. They were obtained in the following way: mass $M$ and radius $R$ of the planets were measured during transits. The first measurements were reported by
Table 1. Parameters for two "Hot Jupiters". The values used for the calculation of the magnetic moments are given in units normalized to Jupiter, while the relative core radius, resulting from Eqs. (6) and (8), is normalized to the planetary radius. The magnetic moment is given for two cases: "tidal locking" (using the value of $\omega$ given above), and "fast rotation" (for the hypothetical case $\omega=\omega_{\mathrm{J}}$ ).

\begin{tabular}{l|cc}
\hline \hline & HD 209458b & OGLE-TR-56b \\
\hline Semimajor axis $r[\mathrm{AU}]$ & 0.045 & 0.0225 \\
\hline Radius $R\left[R_{\mathrm{J}}\right]$ & 1.42 & 1.23 \\
\hline Mass $M\left[M_{\mathrm{J}}\right]$ & 0.69 & 1.45 \\
\hline Rotation rate $\omega\left[\omega_{\mathrm{J}}\right]$ & 0.12 & 0.34 \\
\hline Core density $\rho_{\mathrm{c}}\left[\rho_{\mathrm{c}, \mathrm{J}}\right]$ & 0.24 & 0.78 \\
\hline Relative core radius $r_{\mathrm{c}} / R(6)$ & 0.54 & 0.86 \\
\hline Relative core radius $r_{\mathrm{c}} / R(8)$ & 0.34 & 0.79 \\
\hline $\mathcal{M}\left[\mathcal{M}_{\mathrm{J}}\right]$ (for tidal locking) & $0.005 \ldots 0.10$ & $0.40 \ldots 0.84$ \\
\hline $\mathcal{M}\left[\mathcal{M}_{\mathrm{J}}\right]$ (for fast rotation) & $0.042 \ldots 0.30$ & $1.1 \ldots 1.7$ \\
\hline
\end{tabular}

Henry et al. (2000), Charbonneau et al. (2000) for HD 209458b and Konacki et al. (2003) for OGLE-TR-56b. We adopt the more recent values given by Cody \& Sasselov (2002) for the planet HD 209458b and those given in Torres et al. (2004) for OGLE-TR-56b. The rotation rate $\omega$ was calculated assuming tidal locking (i.e. assuming the orbital period to be equal to the rotation period, see below), taking orbital distances of $0.045 \mathrm{AU}$ and $0.0225 \mathrm{AU}$ (Konacki et al. 2003), respectively. For the core density $\rho_{\mathrm{c}}$, we assume proportionality to the mean density $\bar{\rho}$ of the planet $\left(\rho_{\mathrm{c}} \propto \bar{\rho}=3 M / 4 \pi R^{3}\right)$. Hubbard (1984) shows that for a planet like Jupiter the average density is expected to be proportional to the central density (i.e. the density in the center of the planet). It can be shown that the same proportionality also holds for the core density $\rho_{\mathrm{c}}$, i.e. the density which is found by averaging from the center to $r_{\mathrm{c}}$. More complex models for planetary interiors (Stevenson 1982) show that for Jupiter and Saturn the mean density of the dynamo region, $\rho_{\mathrm{c}}$, is actually very close to the mean density of the planet $\bar{\rho}$ $\left(\rho_{\mathrm{c}} \approx \bar{\rho}=3 M / 4 \pi R^{3}\right)$. The conductivity is assumed to be the same as for Jupiter, i.e. $\sigma=\sigma_{\mathrm{J}}$.

Similarly to Farrell et al. (1999), we compare different scaling relations and show that although the results differ quantitatively, the qualitative picture remains the same. The planetary parameters described above were inserted in Eqs. (1), (3), (4) and (5). Note that scalings (2) and (4) are identical. For the size of the core radius $r_{\mathrm{c}}$, the two approaches of Eqs. (6) and (8) were adopted. Equation (8) typically yields smaller values for $r_{\mathrm{c}}$ and thus also for $\mathcal{M}$ than Eq. (6). Of the eight results (four scalings for the magnetic moment with two models for the core radius each) the smallest and the largest magnetic moments are given in Table 1. Obviously, the results are not identical, but they are approximately of the same order of magnitude. For Saturn the same scaling laws yield a magnetic moment between $\mathcal{M}_{\mathrm{S}}=(0.035 \ldots 0.14) \mathcal{M}_{\mathrm{J}}$, while the observed magnetic moment is $\mathcal{M}_{\mathrm{S}}=0.1 \mathcal{M}_{\mathrm{J}}$. This demonstrates that although the 
scalings relations are not very precise, they do yield reasonable results.

It seems surprising to find magnetic moments that are much smaller than that of Jupiter, especially for the planet HD 209458b. This arises from the much lower rotation rate of the planet. Planets in very close orbits around their host stars (i.e. semi-major axis smaller than $~ 0.1$ AU), like HD 209458b and OGLE-TR-56b, are subject to strong tidal dissipation, leading to gravitational (tidal) locking on a very short timescale (Seager \& Hui 2002). For a hypothetical Jupiter-class exoplanet orbiting the host star at about $0.05 \mathrm{AU}$, the synchronization timescale is approximately $2 \times 10^{6}$ years. For gravitationally locked planets, the rotation period is equal to the orbital period. In such a case, fast rotation of the planet is not possible.

To be precise, "Hot Jupiters" should not be expected to be exactly tidally locked. It is known that thermal atmospheric tides can drive planets away from synchronous rotation (Showman \& Guillot 2002; Correia et al. 2003). The deviation from synchronous rotation was estimated by Showman \& Guillot (2002). They show that the error this effect introduces for $\omega$ could be as large as a factor of 2. Even in this case the rotation rate of "Hot Jupiters" will still be far below that of the giant planets of the solar system.

Equations (1) to (5) show that all of the commonly employed scaling-laws for the planetary magnetic moment yield a magnetic moment rapidly decreasing with decreasing rotation rate. For this reason small magnetic moments are likely for "Hot Jupiters". To demonstrate this effect, the same calculation as above is repeated for the same planetary parameters, with the only difference being that the rotation rate is assumed to be identical to that of Jupiter (i.e. $\omega=\omega_{\mathrm{J}}$ ) rather than obtaining it from the orbital parameters under the assumption of tidal locking. These values are also given in Table 1 . Note that these values do not represent realistic estimates of magnetic moments. They are given to illustrate the effect of tidal locking on the magnetic moment.

The effect of tidal locking on the size of the planetary magnetosphere will be discussed in Sect. 4. Tidal locking will also strongly affect all processes involving planetary magnetic fields or the size of the magnetosphere, such as the generation of radio emission (Farrell et al. 1999; Zarka et al. 2001), the impact of cosmic rays on the planet (Stadelmann et al. 2004) and atmospheric erosion. Implications on atmospheric erosion are discussed in Sect. 5.

\section{Evolution of the stellar wind and XUV flux on solar-like stars}

\subsection{Estimation of the stellar radiation environment}

The change of the stellar radiation in the X-ray and extreme ultraviolet (EUV) wavelength range $(\lambda \leq 1000 \AA)$ and the particle flux of stellar winds play a major role in the evolution of planetary atmospheres. Observations and studies of isotopic anomalies in planetary atmospheres (Lammer et al. 2000; Lammer \& Bauer 2003b), radiative fluxes, stellar magnetic fields, stellar winds of solar-type stars with different ages (Zahnle \& Walker 1982; Ayres 1997; Guinan \& Ribas 2002; Wood et al. 2002) and lunar and meteorite fossil records (Newkirk 1980) indicate that the young Sun underwent a highly active phase after its arrival on the Zero-Age-MainSequence (ZAMS).

This active phase of young solar-like stars lasted about 0.5 to $1.0 \mathrm{Gyr}$ for G-type stars and include continuous flare events, which indicate that the radiation and particle environment was several hundred times more intense for young $G$ stars. The high radiation levels of the young stars are produced by strong magnetic activity. Observational evidence and theoretical models suggest that the young solar-like stars rotate about 10 times faster than the present Sun and have significantly enhanced magnetically generated coronal and chromospheric XUV emission (Keppens et al. 1995; Guinan \& Ribas 2002).

X-UV observations, obtained from solar-like stars with the ASCA, ROSAT, EUVE, FUSE and IUE satellites, cover a range between $1 \AA$ and $3300 \AA$, except for a gap between $400 \AA$ and $920 \AA$, which is a region of very strong interstellar medium absorption. Details of the data sets and the flux calibration procedure employed are provided in Guinan \& Ribas (2002). Full spectral irradiance tables have already been completed inside the so called Sun in Time program for five solar proxies (Dorren \& Guinan 1994).

As discussed above, for the present work we have focused on the spectral range with $\lambda<1000 \AA$, which includes X-rays and EUV. A power-law relationship in the evolution of the XUV flux with the age of G-type stars was found for EK Dra (130 Myr), $\pi^{1}$ UMa (300 Myr), $\kappa^{1}$ Cet (750 Myr), $\beta$ Com (1.6 Gyr) and $\beta$ Hyi (6.7 Gyr). These data show an excellent correlation between the emitted flux and stellar age (Lammer et al. 2003a). This study indicates that the solar XUV flux 2.5 Gyr and 3.5 Gyr ago was about 3 and 6 times higher than today, respectively. Also, the high-energy X-ray flux of the Zero-Age Main Sequence Sun was stronger by up to several hundred times. These results are in general agreement with former studies (Iben 1965; Cohen \& Kuhi 1979; Gaugh 1977; Zahnle \& Walker 1982; Ayres 1997), although some of these studies focused on stars that are not necessarily true solar proxies.

\subsection{Estimation of the stellar wind parameters}

To obtain estimations of the evolution of the stellar wind velocity $v$ and stellar wind density $n$ of solar-like stars, we use the scaling for stellar mass loss provided by Wood et al. (2002) as well as the scaling for the velocity determined by Newkirk (1980), which was also used by Zhang et al. (1993).

The mass loss rates have been estimated by using recent indirect stellar wind observations (Wood et al. 2002). HST high-resolution spectroscopic observations of the H I Lyman- $\alpha$ feature of several nearby main-sequence stars have revealed neutral hydrogen absorption associated with the interaction between the stars' fully ionized coronal winds with the partially ionized local interstellar medium. By modelling the absorption features formed in the astrospheres of these stars, the first empirically estimated coronal mass loss rates for $\mathrm{G}$ and $\mathrm{K}$ main sequence stars were obtained by Wood et al. (2002). 
The mass loss rates were found from a small sample of solar-like stars where astrospheres can be observed. Wood et al. (2002) found that the mass loss rate increases with stellar activity. The observations of younger solar-like stars indicate that the young Sun had a much denser solar wind than today. The correlation between mass loss and X-ray surface flux follows a power law relationship. This relation indicates that the average solar wind density has been up to 1000 times higher during the first 0.1 Gyr after the Sun reached the ZAMS than it is today (at $4.6 \mathrm{Gyr}$ ). The mass loss rate is related to the observed X-ray flux $\phi_{\mathrm{X}}$ of solar-like stars. When the coronal activity is at its maximum, this relation can be written as a power-law (Wood et al. 2002):

$\dot{M} \propto \phi_{\mathrm{X}}^{1.15}$.

The X-ray flux of a cool main sequence sun-like star depends on the stellar rotation period $P_{\text {rot }}$, which is in turn correlated with the star's ages. According to Wood et al. (2002) these relations can be expressed as

$\phi_{\mathrm{X}} \propto P_{\mathrm{rot}}^{-2.9}$

and

$P_{\text {rot }} \propto t^{0.6}$

respectively. In these relations $t$ denotes the time elapsed since the formation of the stellar system. For the present day solar system, $t=4.6$ Gyr. From Eqs. (9) and (10), one obtains a power law relationship for the mass loss rate as a function of rotation period, $\dot{M}\left(P_{\text {rot }}\right)$. Here, a complication occurs. Equation (9) does look like a scaling law for the mass loss, but what is really measured by Wood et al. (2002) is the total ram pressure, i.e. the product of mass loss and solar wind velocity. The mass loss given by Wood et al. (2002) was obtained by assuming a constant velocity $v$. As we try to find scaling relations for both $n$ and $v$, we have to correct for this problem by writing $\dot{M} v$ rather than $\dot{M}$ in Eq. (9). Thus:

$\dot{M} v \propto P_{\text {rot }}^{-3.3}$

To obtain the time dependence, the relation for $P_{\text {rot }}(t)$ is substituted into Eq. (12). In principle, Eq. (11) could be used, but for consistency with the velocity scaling law given below, we take the scaling relation derived by Newkirk (1980):

$P_{\text {rot }} \propto\left(1+\frac{t}{\tau}\right)^{0.7}$

with the time constant $\tau=2.56 \times 10^{7} \mathrm{yr}$ calculated from Newkirk (1980). Combining Eqs. (12) and (13), it is possible to derive a power law formula for the stellar mass loss as:

$\dot{M} v \propto\left(1+\frac{t}{\tau}\right)^{-2.3}$.

On the other hand, the stellar mass loss linearly depends on $v$ and $n$ :

$\dot{M}=\operatorname{Anvm}_{\mathrm{p}}$,

where $A$ is the stellar surface and $m_{\mathrm{p}}$ the mass of the solar wind protons. So, by finding an independent scaling relation

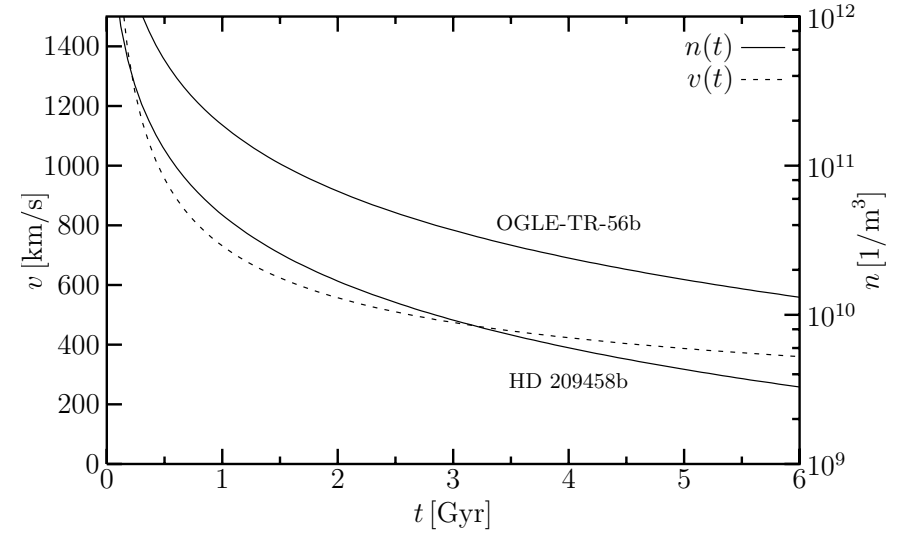

Fig. 1. Time evolution of the stellar wind density (solid lines) and velocity (dashed line) at the orbits of OGLE-TR-56b and HD 209458b.

for the stellar wind velocity, the stellar wind density directly follows from Eq. (14). The time-dependent behaviour of the solar wind velocity can be achieved by (Newkirk 1980; Zhang et al. 1993):

$v=v_{*}\left(1+\frac{t}{\tau}\right)^{-0.4}$.

Note that the argumentation of Newkirk (1980) would lead to a different stellar wind velocity $v(t)$ if a different relation for $P_{\text {rot }}(t)$ was used - such as Eq. (11). Using the mass loss relation Eq. (14) and combining with Eq. (15), we can determine the particle density:

$n=n_{*}\left(1+\frac{t}{\tau}\right)^{-1.5}$

The proportionality constants are determined by the presentday conditions. With $v=400 \mathrm{~km} \mathrm{~s}^{-1}$ and $n=10^{7} 1 / \mathrm{m}^{3}$ for $t=4.6 \mathrm{Gyr}$ and at $r=1 \mathrm{AU}$ (Schwenn 1990), one obtains $v_{*}=$ $3200 \mathrm{~km} \mathrm{~s}^{-1}, n_{*}=2.4 \times 10^{10} 1 / \mathrm{m}^{3}$ (density at $1 \mathrm{AU}$ ). The time constant is $\tau=2.56 \times 10^{7} \mathrm{yr}$ (Newkirk 1980). For distances other than $1 \mathrm{AU}$, Eq. (17) is scaled with a $1 / r^{2}$ dependency. The time variation of $n(t)$ at the orbits of OGLE-TR-56b as well as HD 209458b and $v(t)$ (used for both planets) are shown in Fig. 1. Obviously, for young stellar systems, the stellar wind pressure is much more important than for a star of the age of the solar system. The resulting compression of the magnetosphere by a stellar wind is discussed in the following section.

\section{Qualitative model of the magnetospheric magnetic field}

The magnetosphere of a planet results from the interaction of the stellar wind with the internal planetary magnetic field. The shape of the magnetopause is determined by the pressure equilibrium between the stellar wind ram pressure, the planetary magnetic field pressure and a contribution from the atmospheric pressure.

Since we have no knowledge of the magnetospheric topology of exoplanets, we have to use magnetospheric models to estimate the size and shape of the magnetosphere of such a planet. Several models have been developed during the 
last decades. For example, there are semiempirical models which describe the Earth very well. Analytical models using a potential field ansatz represent a slightly less realistic magnetosphere, but offer the possibility to choose magnetic and geometrical parameters independently. Jordan (1994) and Siscoe (2001) provide good reviews of different magnetospheric models.

In order to model the magnetospheric magnetic field of exoplanets, we used the parametric model that was developed by Voigt (1981) and generalized by Stadelmann et al. (2004). The shape of the magnetosphere is not self-consistent, but described by a semi-infinite cylinder on the nightside and by a half-sphere on the dayside. Both have the same radius $R_{\mathrm{m}}$. The planet is located within the half-sphere at a standoff distance $R_{\mathrm{S}}<R_{\mathrm{m}}$. From observations and models of Jupiter (Joy et al. 2002) and other planets (Voigt et al. 1987; Voigt \& Ness 1990) one can deduce that the ratio between the magnetotail radius and standoff distance is approximately 2 (i.e. one can assume $R_{\mathrm{m}}=2 R_{\mathrm{s}}$ ). The determination of the standoff distance is given below. The magnetic field within the magnetosphere is obtained as a superposition of the planetary magnetic field, which in this case is derived from a dipole moment, and an external magnetic field driven by the magnetopause currents. For the magnetic moment we use the results developed in Sect. 2. Instead of modelling the magnetopause currents, its magnetic fields are given by a scalar potential. This potential meets the boundary condition that the normal component of the magnetospheric magnetic field vanishes at the magnetopause.

To obtain the standoff distance $R_{\mathrm{s}}$, the planetocentric distance of the magnetopause is calculated for the substellar point. The standoff distance can be obtained by the following pressure balance at the substellar point, i.e. for $r=R_{\mathrm{S}}$ :

$p_{\mathrm{s}}=p_{\mathrm{p}}+\frac{1}{2 \mu_{0}}\left(\boldsymbol{B}_{\mathrm{p}}+\boldsymbol{B}_{\mathrm{mc}}\right)^{2}$,

where $p_{\mathrm{s}}$ denotes the stellar wind pressure, $p_{\mathrm{p}}$ is due to the atmospheric pressure at the planet, $\boldsymbol{B}_{\mathrm{p}}$ is the planetary magnetic field and $\boldsymbol{B}_{\mathrm{mc}}$ is the magnetic field of the magnetopause currents. Note, that only a closed magnetosphere is considered.

The stellar wind pressure can be expressed by

$p_{\mathrm{s}}=n m v^{2}$,

where $n$ denotes the number density of the stellar wind protons, $m$ is their mass and $v$ is their velocity.

In Sect. 5 it will be shown that the size of the upper atmosphere of "Hot Jupiters" is of the same order as the magnetosphere. Thus, the contribution of $p_{\mathrm{p}}$ should also be considered. But to demonstrate the effect of atmospheric expansion, we will compare the two cases: a magnetosphere without atmosphere (i.e. $p_{\mathrm{p}}=0$ ) and a neutral atmosphere without the influence of the magnetosphere. For this reason, $p_{\mathrm{p}}$ will not be considered in the following.

As already mentioned in Sect. 2, the internal magnetic field is assumed to arise from a zonal dipole moment. Then the total value $B_{\mathrm{p}}$ of the magnetic field at the magnetopause is derived from a scalar potential:

$B_{\mathrm{p}}=\frac{\mu_{0}}{4 \pi} \frac{\mathcal{M}}{R_{\mathrm{s}}^{3}}$
Table 2. Input parameters and results for the standoff distance $R_{\mathrm{s}}$ The stellar wind parameters $n$ and $v$ are given for a young parent star $(0.5 \mathrm{Gyr})$ and for a parent star of the same age as the Sun $(4.6 \mathrm{Gyr})$. The magnetic moment is taken from the results of Sect. 2 considering tidal locking (tidal lock.) and for comparison the case of fast rotation (fast rot.).

\begin{tabular}{l|cccc}
\hline \hline HD 209458b & $n\left[1 / \mathrm{m}^{3}\right]$ & $v\left[\mathrm{~km} \mathrm{~s}^{-1}\right]$ & $\mathcal{M}\left[\mathcal{M}_{\mathrm{J}}\right]$ & $R_{\mathrm{s}}\left[R_{\mathrm{p}}\right]$ \\
\hline 4.6 Gyr, fast rot. & $4.9 \times 10^{9}$ & 400 & 0.30 & 3.8 \\
4.6 Gyr, tidal lock. & $4.9 \times 10^{9}$ & 400 & 0.10 & 2.6 \\
0.5 Gyr, fast rot. & $1.3 \times 10^{11}$ & 955 & 0.30 & 1.6 \\
0.5 Gyr, tidal lock. & $1.3 \times 10^{11}$ & 955 & 0.10 & 1.1 \\
\hline OGLE-TR-56b & $n\left[1 / \mathrm{m}^{3}\right]$ & $v\left[\mathrm{~km} \mathrm{~s}^{-1}\right]$ & $\mathcal{M}\left[\mathcal{M}_{\mathrm{J}}\right]$ & $R_{\mathrm{s}}\left[R_{\mathrm{p}}\right]$ \\
\hline 4.6 Gyr, fast rot. & $2.0 \times 10^{10}$ & 400 & 1.7 & 6.1 \\
4.6 Gyr, tidal lock. & $2.0 \times 10^{10}$ & 400 & 0.84 & 4.9 \\
0.5 Gyr, fast rot. & $5.0 \times 10^{11}$ & 955 & 1.7 & 2.7 \\
0.5 Gyr, tidal lock. & $5.0 \times 10^{11}$ & 955 & 0.84 & 2.1 \\
\hline
\end{tabular}

As a worst case, the maximum value of the magnetic moment of Table 1 is assumed.

Since the magnetopause currents flow only on the boundary, their magnetic field can be deduced from a scalar potential. For a spherical magnetopause this potential can be described by spherical harmonics. At the substellar point the expression for the magnetic field is similar to the planetary magnetic field in Eq. (20), so the total magnetic field can be written as

$\boldsymbol{B}_{\mathrm{p}}+\boldsymbol{B}_{\mathrm{mc}}=3 \boldsymbol{B}_{\mathrm{p}}=: 2 f_{0} \boldsymbol{B}_{\mathrm{p}}$

Here the form factor is $f_{0}=1.5$ for a spherical magnetosphere. For a more realistic magnetopause shape, but not a sphere, a factor $f_{0}=1.16$ is given in Voigt (1995). This value will be used in the following.

Inserting Eqs. (19)-(21) into the pressure equilibrium of Eq. (18) and neglecting the contribution from the atmospheric pressure $p_{\mathrm{p}}$, as mentioned above, the standoff distance is found to be

$R_{\mathrm{S}}=\left[\frac{\mu_{0} f_{0}^{2} \mathcal{M}^{2}}{8 \pi^{2} n m v^{2}}\right]^{1 / 6}$

Note that the planetary magnetic moment $\mathcal{M}$ is assumed to be constant, so that the time variation of the standoff distance originates only from variations of $v$ and $n$.

Table 2 summarizes the parameters for various configurations as well as the standoff distance resulting from Eq. (22). Note that the age of $4.6 \mathrm{Gyr}$ merely serves as a reference case. The age of the star HD 209458 is probably between 4 and 7 Gyr with 5.2 Gyr as a likely value (Mazeh et al. 2000; Cody $\&$ Sasselov 2002). For OGLE-TR-56 the age is $3 \pm 1 \mathrm{Gyr}$ (Sasselov 2003).

As discussed previously, the shape of the magnetospheres is derived using the parametric model of Stadelmann et al. (2004) with the standoff distances from Table 2 . The resulting magnetospheres are shown in Figs. 2 to 4. 

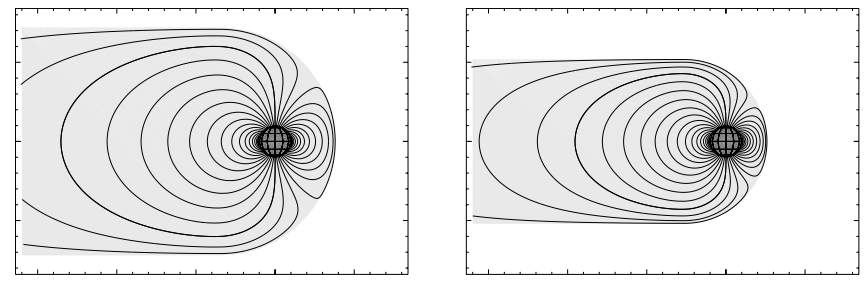

Fig. 2. Magnetosphere of the planet HD 209458b, with field lines. Age of parent star: 4.6 Gyr. The scale is given in planetary radii. Left: fast rotating planet (strong magnetic field). Right: tidally locked planet (weak magnetic field).
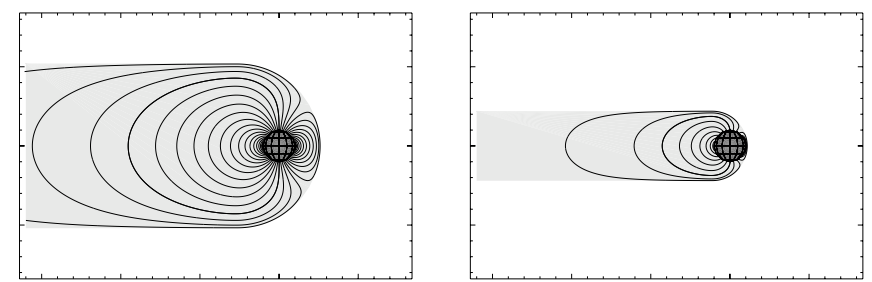

Fig. 3. Magnetosphere of the tidally locked planet HD 209458b, with field lines. The scale is given in planetary radii. Left: age of parent star is $4.6 \mathrm{Gyr}$. Right: age of parent star is $0.5 \mathrm{Gyr}$.
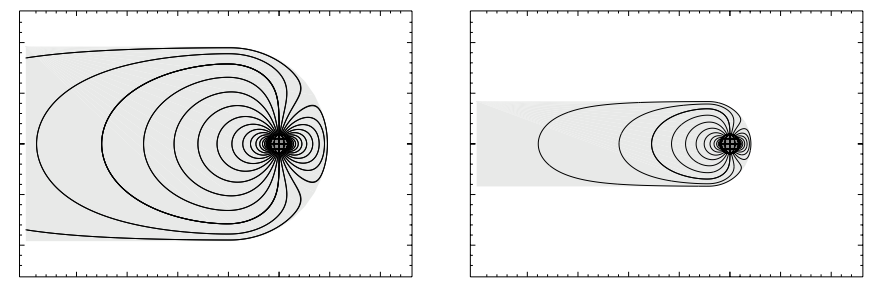

Fig. 4. Magnetosphere of the tidally locked planet OGLE-TR-56b, with field lines. The scale is given in planetary radii. Left: age of parent star is 4.6 Gyr. Right: age of parent star is 0.5 Gyr.

Figure 2 shows the effect of tidal locking on the size of the magnetosphere. As shown in Sect. 2 a tidally locked planet has a significantly reduced magnetic moment, which directly influences the size of the magnetosphere.

Figures 3 and 4 demonstrate the compression of the magnetosphere by the dense stellar wind during the early phase of the stellar evolution. The evolution of the stellar wind with time was discussed in Sect. 3.2.

\section{Atmosphere protection and erosion}

The recent detection of an extended H Lyman- $\alpha$ exosphere of HD 209458b, and its observation based estimated mass loss rate of about $\geq 10^{10} \mathrm{~g} / \mathrm{s}$ (Vidal-Madjar et al. 2003) indicate that the upper atmospheres of "Hot Jupiters" orbiting close to their host stars may be affected by XUV-driven hydrodynamic conditions. Moreover, new HST observations of the heavy atmospheric constituents oxygen and carbon beyond the Roche lobe of HD 209458b by Vidal-Madjar et al. (2004) can be seen as a confirmation that the hydrodynamically driven hydrogen wind also carries heavier species away from the planet.

In a recent study by Lammer et al. (2003a), a scaling relation from solar system planets was used to estimate the exospheric temperature for giant exoplanets at various orbital distances. The scaling relation in that study is based on the assumption of equilibrium between the XUV heat input and downward heat transport by conduction. It was found that large exospheric temperatures in the order of about $10000 \mathrm{~K}$ for hydrogen dominated atmospheres develop, so that a hydrodynamic treatment must be considered (Watson et al. 1981; Lammer et al. 2003a). This results in cooling and a temperature decrease due to large XUV driven loss rates and atmospheric expansion. These exospheric temperature values are in agreement with previous suggestions of Schneider et al. (1998) and Moutou et al. (2001).

Jeans proposed a model of an isothermal atmosphere in diffusive equilibrium that gives accurate thermal atmospheric escape rates. This law is valid as long as the thermal escape parameter

$X=\left(v_{\infty} / v_{0}\right)^{2}$

at the exobase does not reach values much smaller than 1.5 (Öpik 1963). Here $v_{\infty}$ is the escape velocity of the planet and $v_{0}=\left(2 k T_{\infty} / m\right)^{1 / 2}$ is the most probable velocity of the exospheric particles in the exosphere, $T_{\infty}$ is the exospheric temperature, $k$ the Boltzmann constant and $m$ the mass of hydrogen. Lammer et al. (2003a) found that if $X$ reaches values $<3$ diffusive equilibrium no longer applies. For this case the thermal energy exceeds the gravitational potential energy (Öpik 1963; Bauer 1971; Gross 1972; Watson et al. 1981)

$\frac{3}{2} k T_{\infty}>\frac{M m G}{r}$,

where $G$ is the gravitational constant and $M$ is the planetary mass. This leads to the onset of pure hydrogen winds only limited by the incoming XUV flux (Watson et al. 1981; Lammer et al. 2003a). Therefore, the Jeans treatment is not applied for small values of $\mathrm{X}$ because mass loss can no longer be estimated from the Jeans equation. In this case, hydrodynamic conditions must be taken into account. By considering a dynamically expanding non-viscous gas of constant molecular weight, one can use the following model (Watson et al. 1981; Lammer et al. 2003a):

(1) At the distance $r_{0}$, given by

$r_{0}=\frac{G M m}{k T_{0} X_{0}}$,

the gas is bound to the planet and no escape takes place. Here the temperature is fixed at $T_{0}$ corresponding to the effective temperature $T_{\text {eff }}$, and we set $X_{0} \approx 30$ (for which less than $10^{-12}$ of the particles are escaping).

(2) The main XUV-flux is absorbed at a distance $r_{1} \geq r_{0}$, defined by

$r_{1}=\frac{X_{0}}{X_{1}} r_{0}$

where $X_{1}$ is the escape parameter numerically obtained from Eqs. (7) and (8) of Lammer et al. (2003a).

Above $r_{1}$ the optical depth for the stellar XUV-flux is much smaller than 1, and below $r_{1}$ it is larger than 1. Absorption thus 


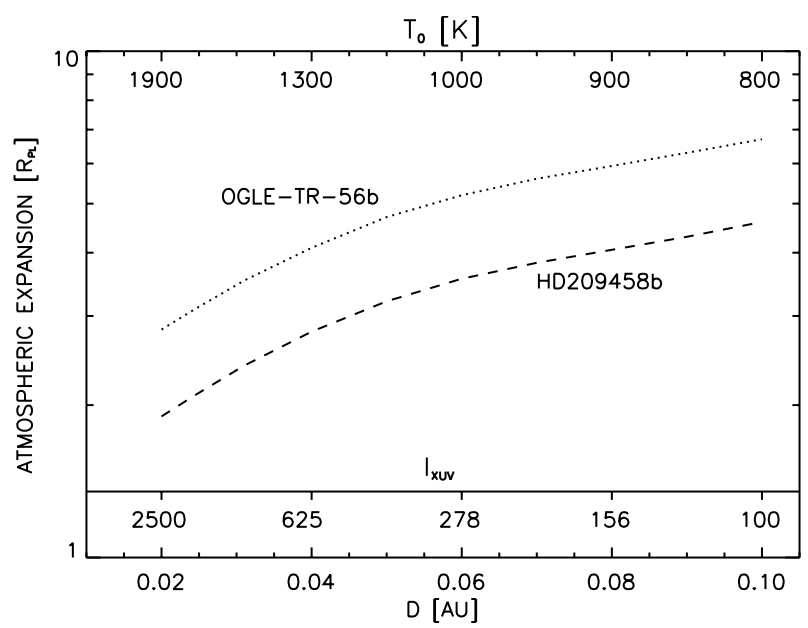

Fig. 5. XUV-driven hydrodynamic expansion of the upper atmosphere for "Hot Jupiters" with the mass of HD 209458b (dashed line) and OGLE-TR-56b (dotted line). Shown: expansion radius $r_{1}$ (in planetary radii) as a function of orbital distance, $T_{0}$ and stellar XUV flux. The expansion depends on the XUV flux, $T_{0}$, orbital distance and thus on the age of the star and also on the escape parameter $X_{0}$, which is taken to be 30. One can see that our calculations of the atmospheric expansion fit well with the Lyman- $\alpha$ radius observed on HD 209458b at about 0.045 AU (Vidal-Madjar et al. 2003).

is important for $r \leq r_{1}$. Because of the hydrodynamic expansion, $r_{0}$ and $r_{1}$ are usually much larger than the altitudes at which the visible opacity is about unity.

By solving simultaneously the equations for the flux of the escaping particles and the escape parameter $X_{1}$, one can calculate the expanded radius $r_{1}$ (Watson et al. 1981; Lammer et al. 2003a). This radius is shown for OGLE-TR-56b and HD 209458b in Fig. 5.

As shown in Fig. 5, HD 209458b has an extended upper atmosphere of about 3 planetary radii at its orbital distance of about 0.045 AU. From Eq. (25) one can also see that for a constant $X_{0}, r_{0}$ moves closer to the planet if $T_{0}$ and the incident XUV flux increases. As a result from Eq. (26), $r_{1}$ may also be closer to the defined planetary radius although the XUVdriven hydrodynamic escape rates increase. Corresponding to its planetary and stellar parameters OGLE-TR-56b, should also have an extended upper atmosphere of about 3 planetary radii at an orbital distance of about 0.0225 AU.

By using the modelled expansion of the upper atmospheres for HD 209458b, a present maximal energy limited mass loss rate of non-ionized hydrogen atoms of about $10^{12} \mathrm{~g} / \mathrm{s}$ was calculated by Lammer et al. (2003a). This is in good agreement with the observational value inferred by Vidal-Madjar et al. (2003). It should be noted that the determined minimal escape rate of non-ionized hydrogen atoms of about $10^{10} \mathrm{~g} / \mathrm{s}$ by VidalMadjar et al. (2003) may be of orders of magnitude higher because of saturation of the absorption line (Vidal-Madjar, personal communication). An estimation of the present mass loss rate for OGLE-TR-56b is $\sim 5 \times 10^{12} \mathrm{~g} / \mathrm{s}$ (Lammer et al. 2003a).

Figure 6 shows the magnetopause distance, where the incoming stellar wind ram pressure equals the estimated magnetospheric pressure of HD 209458b and OGLE-TR-56b without tidal locking (dashed lines) and with tidal locking

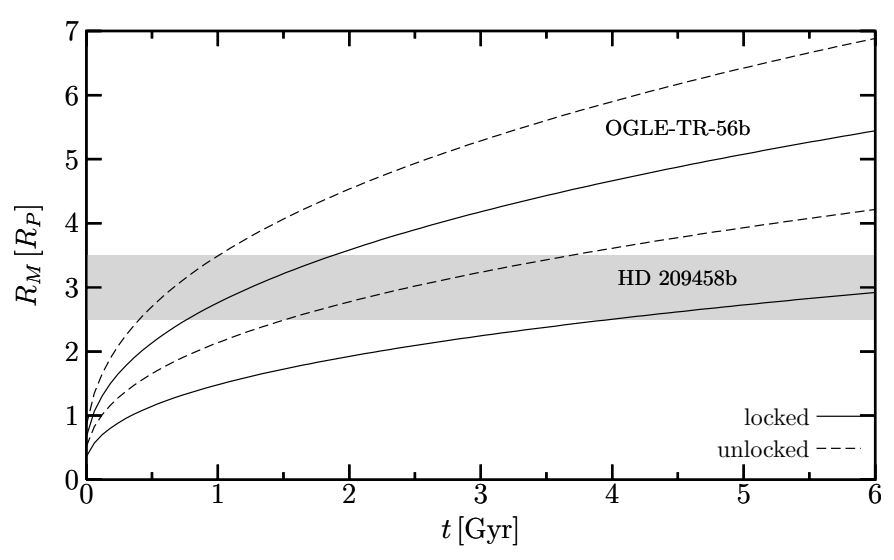

Fig. 6. Standoff distance compared to the size of an expanded atmosphere in planetary radii $\left(1.42 R_{\mathrm{J}}\right.$ for HD $209458 \mathrm{~b}$, and $1.23 R_{\mathrm{J}}$ for OGLE-TR-56b) for HD 209458b and OGLE-TR-56b as a function of time. Tidally locked planets (solid lines) clearly have a smaller magnetosphere than (hypothetical) fast rotating planets (dashed lines). The shaded area shows the distance of the modelled expanded upper atmospheres, inside a range related to observation based uncertainties. It can be seen that the atmosphere is not shielded by the magnetosphere over long periods of time.

(solid lines). As mentioned in Sect. 4, the contribution of the atmosphere to the pressure balance (18) is not taken into account. One can see that both "Hot Jupiters" may not have been protected by their intrinsic magnetic fields at least during the first 0.5 Gyr after their host stars arrived at the ZAMS, even if the effect of tidal locking is ignored. During this early epoch the stellar wind of the young stars was so strong that HD 209458b and OGLE-TR-56b would have had a Venus- or Mars-like interaction between the stellar wind and the atmosphere resulting in the erosion of ionized atmospheric constituents.

If tidal locking is considered for the planet HD 209458b, the magnetic field may even today not protect the atmosphere from the stellar wind and hence from ion loss. The atmosphere of OGLE-TR-56b was not protected by its magnetic field approximately over the first Gyr after its host star arrived on the ZAMS.

The compression of intrinsic planetary magnetic fields of "Hot Jupiters" should enhance the non-thermal ion loss rates caused by ion pick up (Lammer \& Bauer 1991; Lichtenegger et al. 1995; Lichtenegger \& Dubinin 1998; Leblanc \& Johnson 2001, 2002; Lichtenegger et al. 2002) and detached ionospheric clouds (Brace et al. 1982) caused by magnetohydrodynamic plasma instabilities (Arshukova et al. 2003; Penz et al. 2003) at the stellar wind-ionospheric boundaries. Ion loss was not studied by Lammer et al. (2003a) but was estimated by Guillot et al. (1996). For present stellar activity they estimate loss rates of the order of about $10^{10} \mathrm{~g} / \mathrm{s}$ for HD 209458b. However, their study included only photoionized hydrogen and neglected compressed magnetospheres and more complicated ion loss processes triggered by plasma instabilities as observed above the Venus ionopause (Brace et al. 1982; Arshukova et al. 2003; Penz et al. 2003). The application of models developed for the study of the solar wind-ionosphere interaction processes on Venus is beyond the scope of this paper. These models will be applied to "Hot 
Jupiters" in the future, so that the efficiency of ion erosion processes can be studied during periods where these exoplanets may have had a Venus-like stellar wind-interaction.

One should note that the non-thermal loss processes may not give rise to an additional contribution besides the total energy-limited escape rates estimated by the hydrodynamic approach. Rather, it will lead to the ionization of a large part of the escaping gas. The estimation of the ionized population is important for spectroscopic searches for atmospheric species in the stellar spectra of the host star (Moutou et al. 2001).

It was shown by Lammer et al. (2003a) that the original mass of HD 209458b after the exoplanet arrived or originated at the orbital distance of about $0.045 \mathrm{AU}$ may have been about 50\% to $80 \%$ larger, than it is at present. As one can see from Eqs. (6) or (8) a larger mass at the beginning should result in a larger core. According to Eqs. (1)-(5), this results in a larger magnetic moment. Such a larger magnetic moment may have better protected the upper atmospheres against ionospheric erosion processes.

\section{Conclusions}

We have modeled the stellar wind magnetospheric interaction for present and early stages of HD 209458b and OGLE-TR-56b. Our study suggests that because of tidal locking, the magnetic moments of giant exoplanets can be less than one tenth of the value presently observed for Jupiter. Thus the intrinsic magnetic field cannot balance the stellar wind pressure at orbital distances $<0.05 \mathrm{AU}$. We found that because of the expected effect of tidal locking, HD 209458b may have had an ionosphere-stellar wind interaction like Venus or Mars during most of its lifetime. The upper atmosphere of OGLE-TR-56b may have been unprotected during the first Gyr after the host star arrived on the ZAMS.

Estimates of planetary magnetic moments are improved by accounting not only for the planetary mass, but also its radius. This appears to be necessary, as the detected transiting "Hot Jupiters" exhibit larger radii (i.e. lower density) than expected by comparing to the solar system.

The evolution of stellar wind density and stellar wind velocity over the last $4.6 \mathrm{Gyr}$ is discussed. We showed that, due to a much larger stellar wind ram pressure in early stellar periods up to about $0.5 \mathrm{Gyr}$ after the host stars arrived on the ZAMS, "Hot Jupiters" may have not been protected by their intrinsic magnetic fields, even if one neglects the effect of tidal locking. A stellar wind interaction like on Venus would not be possible if "Hot Jupiters" did not have a XUV-driven hydrodynamic expanded upper atmosphere. We found that the stellar wind pressure compresses the magnetosphere to a stand-off distance of about 3 planetary radii. In this case the ionized part of the expanded atmosphere forms a barrier. The calculated expansion distance of the upper atmosphere agrees with the recent observation of an expanded exosphere of HD 209458b (Vidal-Madjar et al. 2003).

As a consequence of hydrodynamic conditions, neutral hydrogen escape rates on "Hot Jupiters" are expected to be in the order of about $\leq 10^{12} \mathrm{~g} / \mathrm{s}$. The dynamics of the outflow of an expanding upper atmosphere against the pressure of the stellar wind may be considered to be similar to a comet's tail (Gross 1972; Schneider et al. 1998; Moutou et al. 2001). Because of the reduced magnetic moment resulting from tidal locking, our study indicates that the upper atmospheres of exoplanets orbiting at close distances around their host stars may be unprotected by their intrinsic magnetic fields over geological periods. Thus their atmosphere and ionosphere will be affected like Venus by different non-thermal ion loss processes such as ion pick up and detached ionospheric clouds triggered by magnetohydrodynamic instabilities. Our study suggests that because of the much larger particle and radiation environment of young solar-like stars, both thermal and ion loss rates from an unprotected atmosphere may have been orders of magnitudes larger during the first Gyr after their host star arrived at the ZAMS. Therefore they should have significantly influenced the evolution of the mass and radius of "Hot Jupiters". The new generation of space observatories like COROT may have the possibility to detect the remaining cores (Rouan et al. 2000) of giant exoplanets orbiting close to their host stars. These planetary cores may develop after the loss of their dense hydrogen atmospheres by thermal and non-thermal loss processes.

Acknowledgements. We would like to thank T. Guillot for very useful comments and suggestions. H. Lammer thanks J. Linsky from the University of Colorado, Boulder, USA for discussions regarding stellar winds and the early solar particle environment. T. Penz acknowledges support by a Paul Urban scholarship from the Institute of Theoretical Physics of the University of Graz, Austria. E. F. Guinan and I. Ribas acknowledge support from NASA/FUSE grants NAG5-10387 and NAG5-12125. W. W. Weiss is supported by the Austrian Ministery of Science (bm:bwk), the Austrian Space Agency (ASA) and the FWF Austrian Science Fund (P14984). Part of this work is supported by the Autrian Science Fund, FWF, under Project P17099-N08.

\section{References}

Arshukova, I. L., Erkaev, N. V., Biernat, H. K., \& Vogl, D. F. 2004, Adv. Space Res., 33, 182

Ayres, T. R. 1997, J. Geophys. Res., 102, 1641

Bauer, S. J. 1971, Nature Phys. Sci., 232, 101

Blackett, P. M. S. 1947, Nature, 159, 658

Blackett, P. M. S. 1952, Phil. Trans. R. Soc. A, 245, 309

Bodenheimer, P., Laughlin, G., \& Lin, D. N. C. 2003, ApJ, 592, 555

Brace, L. H., Theis, R. F., \& Hoegy, W. R. 1982, Planet. Space Sci., 30,29

Burrows, A., Guillot, T., Hubbard, W. B., et al. 2000, ApJ, 534, L97

Busse, F. H. 1976, Phys. Earth Planet. Inter., 12, 350

Charbonneau, D., Brown, T. M., Latham, D. W., \& Mayor, M. 2000, ApJ, 529, L45

Cody, A. M., \& Sasselov, D. D. 2002, ApJ, 569, 451

Cohen, M., \& Kuhi, L. V. 1979, ApJS, 41, 743

Correia, A. C. M., Laskar, J., \& de Surgy, O. N. 2003, Icarus, 163, 1

Curtis, S. A., \& Ness, N. F. 1986, J. Geophys. Res., 91, 11003

Dorren, J. D., \& Guinan, E. F. 1994, The Sun in time: detecting and modelling magnetic inhomogenities on solar-type stars, in The Sun as a Variable Star, ed. J. M. Pap, C. Frölich, H. S. Hudson, \& S. K. Solanki, 206 (Cambridge: Cambridge University Press)

Farrell, W. M., Desch, M. D., \& Zarka, P. 1999, J. Geophys. Res., 104, 14025 
Gough, D. O. 1977, Theoretical predictions of variations of the solar output, in The Solar Output and its Variations, ed. O. R. White (Boulder: University of Colorado Press), 451

Goldreich, P., \& Soter, S. 1966, Icarus, 5, 375

Gross, S. H. 1972, J. Atmos. Sci., 29, 214

Guillot, T., Burrows, A., Hubbard, W. B., Lunine, J. I., \& Saumon, D. 1996, ApJ, 459, L35

Guillot, T., \& Showman, A. P. 2002, A\&A, 385, 156

Guinan, E. F., \& Ribas, I. 2002, in The Evolving Sun and its Influence on Planetary Environments, ASP Conf. Ser., 269, 85

Henry, G. W., Marcy, G. W., Butler, R. P., \& Vogt, S. S. 2000, ApJ, $529, \mathrm{~L} 41$

Hubbard, W. B. 1984, Planetary interiors (New York: Van Nostrand Reinhold Co.)

Iben, Jr. I. 1965, ApJ, 141, 993

Jordan, C. E. 1994, Rev. Geophys., 32, 139

Joy, S. P., Kivelson, M. G., Walker, R. J., Khurana, K. K., \& Russel, C. T. 2002, J. Geophys. Res., 107

Keppens, R., MacGregor, K. B., \& Charbonneau, P. 1995, A\&A, 294, 469

Konacki, M., Torres, G., Jha, S., \& Sasselov, D. D. 2003, Nature, 421, 507

Lammer, H., \& Bauer, S. J. 1991, J. Geophys. Res., 96, 1819

Lammer, H., Stumptner, W., Molina-Cuberos, G. J., Bauer, S. J., \& Owen, T. 2000, Planet. Space Sci., 48, 529

Lammer, H., Selsis, F., Ribas, I., et al. 2003a, ApJ, 598, L121

Lammer, H., \& Bauer, S. J. 2003b, Space Sci. Rev., 106, 281

Leblanc, F., \& Johnson, R. E. 2001, Planet. Space Sci., 49, 645

Leblanc, F., \& Johnson, R. E. 2002, J. Geophys. Res., 107, E2, (5), 1

Lichtenegger, H. I. M., Schwingenschuh, K., Dubinin, E. M., \& Lundin, R. 1995, J. Geophys. Res., 100, 21,659

Lichtenegger, H. I. M., \& Dubinin, E. M. 1998, Earth Planets Space, 50,445

Lichtenegger, H. I. M., Lammer, H., \& Stumptner, W. 2002, J. Geophys. Res., 107 (A10), 1279

Mazeh, T., Naef, D., Torres, G., et al. 2000, ApJ, 532, L55

Mizutani, H., Yamamoto, T., \& Fujimura, A. 1992, Adv. Space Res., 12,265

Moutou, C., Coustenis, A., Schneider, J., et al. 2001, A\&A, 371, 260

Nellis, W. J. 2000, Planet. Space Sci., 48, 671

Newkirk, Jr. G. 1980, Geochi. Cosmochi. Acta Suppl., 13, 293

Öpik, E. J. 1963, Geophys. J. Roy. Astron. Soc., 7, 490
Penz, T., Erkaev, N. V., Biernat, H. K., et al. 2004, Planet. Space Sci., accepted

Rouan, D., Baglin, A., Copet, E., et al. 2000, Earth, Moon, and Planets, 81, 79

Sano, Y. 1993, J. Geomag. Geoelectr., 45, 65

Sasselov, D. D. 2003, ApJ, 596, 1327

Schneider, J., Rauer, H., Lasoto, S. P., Bonarrola, S., \& Chassefiére, E. 1998, in Brown Dwarfs and Extrasolar Planets, ASP Conf. Ser., 134,241

Schwenn, R. 1990, in Physics of the Inner Heliosphere (Berlin: Springer-Verlag), 99

Seager, S., \& Hui, L. 2002, ApJ, 574, 1004

Shkolnik, E., Walker, G. A. H., \& Bohlender, D. A. 2003, ApJ, 597, 1092

Showman, A. P., \& Guillot, T. 2002, A\&A, 385, 166

Siscoe, G. L. 2001, in Space weather, ed. P. Song, H. J. Singer, \& G. L. Siscoe (Geophysical Monograph), 125, 211

Stadelmann, A., Vogt, J., Glassmeier, K.-H., Kallenrode, M.-B., \& Voigt, G.-H. 2004, Ann. Geophysicae, submitted

Stevenson, D. J. 1982, Ann. Rev. Earth Planet. Sci., 10, 257

Stevenson, D. J. 1983, Rep. Prog. Phys., 46, 555

Torres, G., Konacki, M., Sasselov, D. D., \& Jha, S. 2004, ApJ, in press

Vidal-Madjar, A., des Etangs, A. L., Désert, J.-M., et al. 2003, Nature, 422, 143

Vidal-Madjar, A., Désert, J.-M., Lecavelier des Etangs, A., et al. 2004, ApJ, 604, L69

Voigt, G.-H. 1981, Planet. Space Sci., 29, 1

Voigt, G.-H. 1995, in Handbook of atmospheric electrodynamics, ed. H. Volland, Vol. II (CRC Press), 333

Voigt, G.-H., Behannon, K. W., \& Ness, N. F. 1987, J. Geophys. Res., 92,15337

Voigt, G.-H., \& Ness, N. F. 1990, Geophys. Res. Lett., 17, 1705

Wood, B. E., Müller, H.-R., Zank, G. P., \& Linsky, J. L. 2002, ApJ, 574, 412

Watson, A. J., Donahue, T. M., \& Walker, J. C. G. 1981, Icarus, 48, 150

Zahnle, K. J., \& Walker, J. C. G., 1982 Rev. Geophys. Space Phys., 20,280

Zarka, P., Treumann, R. A., Ryabov, B. P., \& Ryabov, V. B. 2001, Astrophys. Space Sci., 277, 293

Zhang, M. G. H., Luhmann, J. G., Bougher, S. W., \& Nagy, A. F. 1993, J. Geophys. Res., 98, 10915 